\title{
Anne Pezet, Éric Pezet, La société managériale. Essai sur les nanotechnologies de l'économique et du social
} Montreuil, La ville brûle, 2010

\section{Yuri Biondi}

\section{OpenEdition}

\section{Journals}

Édition électronique

URL : http://journals.openedition.org/travailemploi/5800

DOI : 10.4000/travailemploi.5800

ISSN : $1775-416 \mathrm{X}$

Éditeur

DARES - Ministère du Travail

\section{Édition imprimée}

Date de publication : 15 septembre 2012

Pagination : 142-143

ISSN : 0224-4365

\section{Référence électronique}

Yuri Biondi, «Anne Pezet, Éric Pezet, La société managériale. Essai sur les nanotechnologies de l'économique et du social », Travail et Emploi [En ligne], 131 | 2012, mis en ligne le 01 décembre 2012 consulté le 22 septembre 2020. URL : http://journals.openedition.org/travailemploi/5800 ; DOI https://doi.org/10.4000/travailemploi.5800

Ce document a été généré automatiquement le 22 septembre 2020

(c) Direction de l'animation de la recherche, des études et des statistiques (Dares) 


\section{Anne Pezet, Éric Pezet, La société managériale. Essai sur les nanotechnologies de l'économique et du social}

Montreuil, La ville brûle, 2010

\section{Yuri Biondi}

\section{RÉFÉRENCE}

Anne Pezet, Éric Pezet, La société managériale. Essai sur les nanotechnologies de l'économique et du social, Montreuil, La ville brûle, 2010, 141 p.

\section{Vers un management à visage humain?}

2 Spectateur intéressé du débat sociopolitique de son temps, le poète italien Giacomo Leopardi soulignait déjà au début $\mathrm{du} \mathrm{XIX}^{\mathrm{e}}$ siècle la foi des Anglais dans le rôle des "machines", et celle des Français dans celui des "écrits politiques ", comme instruments de progrès social ${ }^{1}$. Dans cette perspective, le livre que nous présentons pourrait être perçu comme un écrit politique de tradition française, consacré aux «nanotechnologies de l'économique et du social»; ces dernières constituent les « machines de gestion » (selon l'expression de Jacques Girin citée p. 13) qui prolongent les usages de la technologie de la sphère matérielle à la sphère sociale.

Les auteurs s'appliquent à montrer la place et le rôle de ces nouvelles "machines diaboliques » (satanic mills) qui sont à la fois portées par et porteuses d'une autre révolution industrielle: celle du «gouvernement managérial », qui dominerait nos économie et société. La démonstration des auteurs suit trois étapes : d'abord, définir ces techniques et dispositifs de gestion souvent ignorés et méconnus par les décideurs, les chercheurs et, plus généralement, la société civile. Ensuite, mettre en évidence leur importance dans les changements organisationnels qui caractérisent les évolutions 
récentes dans le secteur public comme dans le secteur privé. Enfin, engager une appréhension accrue et un véritable " débat démocratique » sur ce gouvernement.

D'après les auteurs, les dispositifs de gestion sont de «fantastiques instruments d'observation de la matière sociale» (p.23) et se caractérisent par leur "pouvoir d'intervention» (ibid.), leur capacité à "atteindre les individus individuellement et collectivement » (ibid.). Les auteurs les définissent selon les critères de Michel Foucault (cité notamment p.16) qui relie tout dispositif, d'une part à un jeu de pouvoirs, et, d'autre part, à des «bornes de savoirs » qui supportent ces pouvoirs et sont supportés par eux. Cui prodest ? ${ }^{2}$ Le gouvernement managérial de notre temps serait promu par certains dirigeants des grandes entreprises (et institutions financières), justifié par des auteurs académiques, vendu par des consultants, et prescrit par des enseignants (pp. 54-63). De plus, ces dispositifs de gestion sont développés souvent par des instances de régulation "privatisée", qui sont mentionnées par ailleurs (p.39) et auraient certainement mérité un approfondissement ultérieur de la part des auteurs.

Plusieurs exemples de dispositif sont donnés, notamment le procédé d'actualisation financière utilisé dans l'évaluation des investissements (p. 28 et suivantes; p. 87 et suivantes); les normes comptables internationales dictées par l'International Accounting Standards Board (IASB; p. 64 et suivantes); les normes qualité (p. 67 et suivantes); les batteries d'indicateurs de performance publique issus de la LOLF (Loi organique relative aux lois de finances du 25 janvier 2006, p. 68 et suivantes). Une attention particulière est portée à la détermination des coûts, dont la réduction motive des politiques d'entreprise aux conséquences sociétales lourdes, mais dont les fondements restent méconnus, voire douteux (p. 86 et suivantes). Une analyse de la «valeur actionnariale» et de ses mécanismes de mise en œuvre aurait rendu l'investigation socioéconomique des politiques d'entreprise encore plus actuelle et percutante ${ }^{3}$. À ce sujet, les auteurs abordent également l'impact des études de marché sur les rapports sociaux et la conception de soi (la référence est ici aux études de l'historien allemand Christoph Conrad, p. 100 et suivantes), en négligeant toutefois d'analyser la portée et la signification des stratégies et des dépenses publicitaires dans la période plus récente. D'après les auteurs, le gouvernement managérial basé sur ces dispositifs de gestion caractérise les grandes bureaucraties publiques et privées, dans une survivance puissante de l'illusion weberienne quant à l'efficience et à la neutralité de ce mode d'organisation technocratique (au sens de John Kenneth Galbraith) : « Après la Seconde Guerre mondiale, le management est apparu comme un moyen d'échapper aux idéologies et un gouvernement managérial a semblé pouvoir se substituer au gouvernement politique»(pp.45-46). Ce renouveau inattendu de la «planification soviétique » (p. 70) dépasserait même la dimension organisationnelle et sociale pour s'emparer de la dimension personnelle et culturelle dans un processus de " subjectivation » (selon la formule de Foucault citée p. 31) et d'" ascèse » (ibid.). Aucun parallèle n'est tenté cependant avec d'autres analyses anciennes et récentes, à commencer par l'« aliénation", d'empreinte marxienne, ou la dépersonnalisation. La critique de Michel Crozier quant aux failles, à l'inefficience et à la capture par le pouvoir du mode d'organisation bureaucratique est également ignorée 4 .

6 Enfin, le fonctionnement d'ensemble de ces dispositifs de gestion suggère aux auteurs l'émergence d'une "solidarité technique " (selon la définition de Gilbert Simondon citée p. 79). Il montre également l'évidence d'une solidarité mécanique au sens d'Émile Durkheim, qui pourrait sans doute devenir une solidarité voulue et consciente si ces 
dispositifs étaient reformulés et mis en œuvre dans une perspective différente. Ils pourraient sans doute servir l'intérêt commun et général, au lieu de profiter seulement à certains pouvoirs (notamment financiers) dominant temporairement le jeu social et ses «bornes de savoir», selon la formule de Michel Foucault retenue par les auteurs" D'où l'engagement des auteurs pour un "débat démocratique » sur ces dispositifs et une "action politique " au sens large, incluant "la moralisation et la régulation " (p.116). À leur avis, cette action devrait être portée surtout par "les acteurs des organisations, entreprises, administrations ou associations", et être destinée à « repenser les usages [des dispositifs de gestion], [...] prévenir les effets négatifs et [...] opérer une vigilance de tous les instants afin de les éviter» (p.114). La référence est ici à Pierre-Joseph Proudhon qui, en distinguant entre technographie et économie politique, invite à penser et discuter l'articulation entre ces deux dimensions (pp. 76-78). D'après les auteurs, la «vulgate ultralibérale » a fait pendant avec «les savoirs et techniques du gouvernement managérial»(p.118), laissant l'individu dépourvu aussi bien de sa dimension collective et sociale que de sa dimension individuelle et personnelle. À leur avis, une action véritablement politique développée par la recherche, l'enseignement et la formation, les relations professionnelles - pourra alors aider à sortir de l'impasse engendrée par ce gouvernement managérial. En fait, les auteurs n'indiquent guère quels propos ou quelles solutions cette action devrait envisager : serait-il temps de renverser l'intuition chère à Michel Foucault et de réguler enfin les organisations plutôt que les individus ? Quels dispositifs de gestion serviraient à cet univers organisationnel et social métamorphosé ? Comme le voudrait le poète, cette action fera sans doute appel à la liberté des individus, en remettant les organisations sous leur égide, dans un cadre institutionnel capable d'assurer également la solidarité et l'équité du système socioéconomique. Car, comme le suggère le physicien Richard Feynmann (cité p. 24), il y a plein d'espace tout au fond de la matière (qu'elle soit naturelle ou sociale), et plein de marges de liberté, rajouterions-nous.

En conclusion, cet ouvrage introduit les lecteurs à l'analyse socio-économique des instruments de gestion. Bien que certains terrains et implications auraient pu être approfondis davantage, notamment la valeur actionnariale, les dépenses de publicité et de lobbying, ainsi que les retombées en termes de gouvernance, responsabilité sociétale et régulation, il aidera les étudiants de sciences économiques et de gestion à s'initier et à débuter un processus de compréhension et d'appropriation, que les auteurs appellent de leurs vœux pour l'ensemble des acteurs concernés par ces dispositifs.

\section{NOTES}

1. Giacomo Leopardi, Canto XXII, Palinodia, al marchese Gino Capponi, composé à Naples en 1835.

2. À qui profite le crime?

3. A ce sujet, nous renvoyons à Michel Aglietta et Antoine Rebérioux, Les dérives du capitalisme financier, Paris, Albin Michel, 2004. 
4. Nous mentionnerons ici de Michel Crozier : Le Phénomène bureaucratique, Paris, Le Seuil, coll. «Points et Essais », 1964 ; On ne change pas la société par décret, Paris, Fayard, 1979 ; État moderne, État modeste. Stratégies pour un autre changement, Paris, Fayard, 1986; À quoi sert la sociologie des organisations ?, Paris, Arslan, 2000.

5. Voir notamment Michel Foucault, L'archéologie du savoir, Paris, Gallimard, 1969, pp. 68-71.

6. Dans cet esprit, voir le cours donné par Michel Foucault au Collège de France, désormais publié : Naissance de la biopolitique. Cours au Collège de France, 1978-1979, Paris, Gallimard, 2004.

\section{AUTEURS}

\section{YURI BIONDI}

Cnrs - ESCP Europe 